\title{
Diverticulitis Aguda del Colon Izquierdo, Nivel de Consenso y de Aplicación de las Guías de Práctica Clínica Entre Miembros de la SACP Trabajo a Miembro Titular
}

\author{
Diego Hernán Barletta \\ Sanatorio del Oeste, sede Ituzaingó. Buenos Aires, Argentina
}

\begin{abstract}
RESUMEN
Contexto y antecedentes: La elaboración e implementación de guías clínicas pretende brindar ayuda en la toma de decisiones respecto de un determinado problema de salud, sintetizando en forma de recomendaciones la mejor evidencia disponible. Con respecto a la diverticulitis aguda, pese a la gran cantidad de guías que se han elaborado, aún hoy se observa un bajo nivel de consenso en varios aspectos de su manejo.

Objetivos: Este trabajo representa la primera encuesta que mide el nivel de consenso y la aplicación de guías internacionales sobre el manejo de la diverticulitis aguda entre los miembros de la SACP o entre cualquier otra agrupación médica a nivel nacional.

Métodos: Se distribuyó una encuesta online a 313 miembros de la SACP, las respuestas se recolectaron en un período de 2 meses.

Resultados: La encuesta obtuvo una tasa de respuesta de 19,5\%. Solo 17 enunciados superaron el corte de $70 \%$ de nivel de consenso. De ellos, 11 enunciados estuvieron de acuerdo mientras que 2 enunciados estuvieron en desacuerdo con las recomendaciones de las guías internacionales y en los 4 enunciados restantes no pudo valorarse la concordancia debido a falta de respuesta univoca en las guías.

Conclusiones: La amplia heterogeneidad en el manejo de la diverticulitis aguda entre los miembros de la SACP junto con la baja evidencia de los trabajos científicos, la pobre metodología empleada en las guías internacionales y la necesidad de contar con datos locales sobre costos y preferencias, hacen evidente la necesidad que en nuestro ámbito se comience a investigar en forma sistematizada para lograr una guía y consenso nacional.
\end{abstract}

Palabras clave: Diverticulitis Aguda; Guías Clínicas; Nivel de Consenso; Encuesta

\begin{abstract}
Background: Construction and elaboration of clinical guidelines aim to provide help to decision-taking process about a specific health issue, synthesizing the best available evidence in the form of recommendations. About acute diverticulitis, although many guidelines were published in the last years, even now, there is a low consensus level in many aspects of its management.

Aim: This survey is the first one that measures the consensus level and the application of international guidelines, about acute diverticulitis, within the SACP members, or any other medical society in Argentina.

Methods: An online survey was distributed to 313 SACP members; the responses were collected over two months.

Results: The response rate obtained by the survey was 19,5\%. Only 17 statements passed the consensus level of $70 \%, 11$ of them agreed and 2 disagreed with the recommendations of the international guidelines, in the last 4 statements comparison was impossible because the guidelines lacked one single response.

Conclusions: The large heterogeneity in the management of acute diverticulitis within the SACP members, along with the low evidence of the scientific publications, the poor methodology used in the guidelines, and the need of local data about costs and preferences, clearly demonstrates the necessity to start to investigate in a systematic way, in order to achieve a national guideline and consensus about acute diverticulitis.
\end{abstract}

Keywords: Acute Diverticulitis; Guidelines; Consensus Level; Survey

\section{INTRODUCCIÓN}

Con el fin de estandarizar el diagnóstico y tratamiento de la diverticulitis aguda se han elaborado múltiples guías y consensos. A pesar de ello, persiste una gran incertidumbre terapéutica debido a que casi la totalidad de las recomendaciones tienen moderado a bajo nivel de evidencia. Este hecho se hace evidente en varias encuestas realizadas

El autor del trabajo no presenta conflicto de interés.

Diego Hernán Barletta

barlettadie@gmail.com

Recibido: Septiembre de 2019. Aceptado: Noviembre de 2019. a nivel regional e internacional..$^{1-4}$

A la fecha de elaboración del presente trabajo, no existen guías publicadas por ninguna entidad médica nacional respecto del manejo de la diverticulitis aguda, así como tampoco ningún estudio que valore entre los miembros de la Sociedad Argentina de Coloproctología (SACP) el nivel de consenso ni la aplicación de las recomendaciones publicadas por las guías internacionales. Con este objetivo, se realizó una encuesta entre sus miembros que incluyó los escenarios clínicos y recomendaciones expresadas en las guías de práctica clínica y consensos publicados en los últimos 10 años. Cabe mencionar que el presente tra- 
bajo se limita exclusivamente a la diverticulitis aguda del colon izquierdo (DAci) por ser la forma de presentación predominante en los países occidentales.

\section{MATERIALESY MÉTODOS}

Se realizó en la base de datos MEDLINE/PUBMED una búsqueda de las guías, consensos y encuestas sobre diverticulitis aguda publicadas en idioma inglés durante los últimos 10 años al mes de marzo 2019. Para la creación de la encuesta se utilizaron como modelo las guías clínicas y guías incluidas en consensos. En cambio, las encuestas y aquellos consensos que no incluían guías solo se utilizaron para engrosar el marco teórico. Se hallaron 6 guías internacionales ${ }^{5-10}$ y a partir de los enunciados y las recomendaciones vertidas en ellas se creó una encuesta que la SACP difundió, mediante correo electrónico con link a la plataforma google forms, entre 313 miembros titulares y adherentes. La encuesta se organizó en forma de escenarios clínicos con el fin de evitar errores de interpretación en la taxonomía empleada, la cual se describe a continuación para facilitar la descripción de los hallazgos.

Se llama diverticulitis aguda a la presencia de un proceso inflamatorio en los divertículos colónicos. Se la clasifica como:

1. diverticulitis aguda complicada (DAC) cuando se presenta como abscesos, peritonitis, fístula o estenosis, $y$

2. diverticulitis aguda no complicada (DANC) cuando solo afecta la pared colónica.

Si bien existen múltiples clasificaciones, se utilizó la clasificación de Hinchey modificada por Vasvary y colaboradores en $1999 .{ }^{11}$ Como clasificación tomográfica se eligió a la de WSES acute diverticulitis working group por sus cualidades descriptivas, ya que agrupa otras clasificaciones previas y agrega otros hallazgos tomográficos no tenidos previamente en cuenta. ${ }^{12}$

El nivel de consenso se expresó como el porcentaje alcanzado por cada respuesta a cada pregunta. De modo arbitrario se tomó como un nivel aceptable de consenso cuando una respuesta fue elegida por el $70 \%$ o más de la población encuestada, quedando exceptuadas las medidas para prevenir las recurrencias que se midieron como promedio por tratarse de una escala numérica.

Se incorporaron además 2 guías clínicas publicadas con posterioridad a la confección de la encuesta ${ }^{13,14}$ debido a TABLA 1: SEXO, EDAD Y EXPERIENCIA la importancia de las entidades que las elaboraron y haber sido realizadas con la evidencia científica más reciente. A partir de las 8 guías publicadas se realizó una síntesis de sus recomendaciones que se utilizó para medir de forma cualitativa su aplicación por parte de la población encuestada.

\section{Estadística}

Por tratarse de un estudio descriptivo transversal solo se calcularon porcentajes, promedios y rangos ya que los resultados no fueron comparados con ninguna otra población o momento histórico.

\section{RESULTADOS}

La encuesta se llevó a cabo de mayo a julio de 2019. La tasa de respuesta fue de 19,5\% (61/313), la cual, aunque no es significativa, sí es aceptable por tratarse de una encuesta online que es la modalidad de encuesta que presenta las menores tasas de respuesta. ${ }^{15-17}$ En las tablas 1 a 3 se agrupan los datos de los encuestados. En resumen, el 88,5\% fueron hombres con una edad promedio de 46,4 años y casi 15 años de ejercicio profesional como proctólogos. CABA fue el lugar de formación de más de $2 / 3$ de los encuestados y junto con GBA representan el 61,6\% del lugar de trabajo de la muestra. Respecto de la experiencia, el $48,3 \%$ realizó más de 100 procedimientos colorrectales laparoscópicos y el 47,5\% tiene una dedicación exclusiva como proctólogo.

En cuanto al diagnóstico, el 82\% de los encuestados consideró necesario realizar estudios complementarios aun ante un escenario de DANC con mínima respuesta inflamatoria y ausencia de comorbilidades. El 18\% que no los indica rutinariamente solo los indicarían en presencia de:

1. fiebre (100\%),

2. inmunocompromiso $(90,9 \%)$,

3. comorbilidades $(63,6 \%)$,

4. alteraciones del ritmo evacuatorio $(36,4 \%)$ o

5. edad mayor de $60(18,2 \%)$.

Las imágenes fueron los estudios más solicitados en todos los escenarios (tabla 4). De ellos, la TC fue elegida por el 78,7\% considerándola como el estudio más importante (tabla 5). La gran mayoría de los encuestados la solicita con contraste oral y endovenoso $(72,1 \%$ y $88,5 \%$ respectivamente). De los marcadores serológicos el más usado fue el recuento de leucocitos (100\%), seguido por la eritrosedimentación $(36,1 \%)$ y la proteína C reactiva $(31,1 \%)$.

En la tabla 6 se agrupan los tratamientos elegidos según

\begin{tabular}{lcccccc} 
& $\mathrm{N}$ & $\%$ & Edad promedio & Rango & Años de ejercicio como proctólogo & Rango \\
Masculino & 54 & 88,5 & 47 & $30-69$ & 15,4 & $0-40$ \\
Femenino & 7 & 11,5 & 41,9 & $30-48$ & 10,6 & $0-18$ \\
\hline Total & 61 & 100 & 46,4 & $30-69$ & 14,9 & $0-40$
\end{tabular}


hallazgos tomográficos. El manejo ambulatorio fue indicado solo por el 23\% de los encuestados en DANC (estadio 0) y la gran mayoría escogió la internación y antibiótico terapia EV tanto en estadio 0 como en Ia. Respecto del tratamiento de los abscesos, en esta clasificación el estadio Ib usa

TABLA 2: DISTRIBUCIÓN GEOGRÁFICA SEGÚN FORMACIÓN Y LUGAR DE TRABAJO

\begin{tabular}{lcccc} 
Región & $\begin{array}{c}\text { Lugar de } \\
\text { formación } \\
\mathrm{n}=61(\%)\end{array}$ & \multicolumn{3}{c}{$\begin{array}{c}\text { Lugar de } \\
\text { trabajo } \\
\mathrm{n}=60(\%)\end{array}$} \\
CABA & 41 & $67,20 \%$ & 23 & $38,30 \%$ \\
GBA & 7 & $11,50 \%$ & 14 & $23,30 \%$ \\
\hline PAMPEANA & 4 & $6,60 \%$ & 9 & $15,00 \%$ \\
PATAGÓNICA & 2 & $3,30 \%$ & 6 & $10,00 \%$ \\
NOROESTE & 3 & $4,90 \%$ & 5 & $8,30 \%$ \\
NORESTE & 2 & $3,30 \%$ & 3 & $5,00 \%$ \\
CUYO & 0 & $0,00 \%$ & 0 & $0,00 \%$ \\
EXTERIOR & 2 & $3,30 \%$ & $\mathrm{Nc}$ & $\mathrm{Nc}$
\end{tabular}

Nc: no corresponde

TABLA 3: ENTRENAMIENTO LAPAROSCÓPICO Y DEDICACIÓN A LA ESPECIALIDAD

\begin{tabular}{lccccc}
$\begin{array}{l}\text { Cantidad de } \\
\text { procedimien- }\end{array}$ & $\begin{array}{l}\mathrm{n}=60 \\
\text { tos laparos- } \\
\text { cópicos colo }\end{array}$ & \multicolumn{5}{c}{$\begin{array}{c}\text { \% de su } \\
\text { trabajo } \\
\text { dedicado } \\
\text { rectales }\end{array}$} & $\begin{array}{c}\mathrm{n}=61 \\
\text { a la proc- } \\
\text { tología }\end{array}$ \\
$>100$ & 29 & $48,30 \%$ & $>90 \%$ & 29 & $47,50 \%$ \\
$75-99$ & 5 & $8,30 \%$ & $76-90 \%$ & 12 & $19,70 \%$ \\
$50-74$ & 6 & $10,00 \%$ & $51-75 \%$ & 11 & $18,00 \%$ \\
$25-49$ & 7 & $11,70 \%$ & $26-50 \%$ & 8 & $13,10 \%$ \\
$<25$ & 13 & $21,70 \%$ & $<26 \%$ & 1 & $1,60 \%$
\end{tabular}

TABLA 4: ESTUDIOS COMPLEMENTARIOS SEGÚN ESCENARIO CLÍNICO

\begin{tabular}{|c|c|c|c|}
\hline $\begin{array}{l}\text { Tipo de estudio y } \\
\text { escenario }\end{array}$ & $\begin{array}{l}\text { DANC } \\
\sin \text { SIRS }\end{array}$ & $\begin{array}{c}\text { DANC con } \\
\text { SIRS, abscesos }\end{array}$ & $\begin{array}{l}\text { Perito- } \\
\text { nitis }\end{array}$ \\
\hline Imágenes & $96,70 \%$ & $98,40 \%$ & $83,60 \%$ \\
\hline $\begin{array}{l}\text { marcadores rta. } \\
\text { inflamatoria }\end{array}$ & $42,60 \%$ & $75,40 \%$ & $57,40 \%$ \\
\hline Colonoscopía & $0 \%$ & $1,60 \%$ & $0 \%$ \\
\hline Cirugía de urgencia & Nc & $\mathrm{Nc}$ & $50,80 \%$ \\
\hline
\end{tabular}

Nc: no corresponde, no se dio esa opción para esa pregunta.

TABLA 5: ESTUDIOS POR IMÁGENES SOLICITADOS

\begin{tabular}{lcccc} 
Como se lo solicitó & Único & Con otros estudios & $\mathrm{n}=61$ & $\%$ \\
\hline TC de abdomen y pelvis & 28 & 20 & 48 & 78,7 \\
Ecografía & 2 & 19 & 21 & 34,4 \\
Rx abdomen & 0 & 19 & 19 & 31,1 \\
TC luego de Rx y eco negativas & $\mathrm{Nc}$ & $\mathrm{Nc}$ & 11 & 18 \\
\hline RNM si TC está contraindicada & 0 & 7 & 7 & 11,5 \\
RNM & 0 & 0 & 0 & 0
\end{tabular}

Nc: no corresponde, no se dio esa opción para esa pregunta. como corte los $4 \mathrm{~cm}$ de diámetro. Debido a que existe controversia en la literatura si este límite debería ubicarse en los $3 \mathrm{~cm}$ y a fin de evaluar el accionar de la población en la zona de conflicto, se creó un escenario con un absceso de $3,5 \mathrm{~cm}$ en donde un 26,2\% de los encuestados indicó drenaje percutáneo con antibióticos. En estadio IIa el drenaje con antibióticos fue indicado por el 63,9\%, y sin antibióticos por casi el $20 \%$. Ante peritonitis el $59 \%$ indica resección quirúrgica mientras que el $41 \%$ lavado peritoneal laparoscópico. En las tablas 7 y 8 se muestra la indicación de analgésicos y antibióticos en manejo ambulatorio. El manejo de las fallas terapéuticas y el tipo de resección indicada en cada escenario se muestra en las tablas 9 y 10. Respecto de la cirugía de control de daños en caso de inestabilidad hemodinámica, el $44,3 \%$ la menciona como su primera opción, el $47,5 \%$ la reserva solo si no se logra controlar el estado hemodinámico durante la cirugía y el 8,2\% nunca la utiliza.

Los detalles técnicos de la resección quirúrgica electiva que alcanzaron consenso fueron la preparación mecánica, la resección hasta márgenes de tejido sano de colon y recto, el abordaje laparoscópico y la evaluación de fuga de la anastomosis (tabla 11).

En cuanto a la indicación electiva de colonoscopía y de resección quirúrgica (tablas 12 y 13), solo alcanzó consenso la colonoscopía luego del drenaje percutáneo. Las indicaciones para prevenir recurrencias se muestran en la tabla 14.

Con el fin de comparar los resultados de la encuesta se realizó una síntesis de las recomendaciones de las guías publicadas en los últimos años que se muestra en los gráficos 1 a 3 .

Solo 17 enunciados superaron el corte de $70 \%$ de nivel de consenso. De ellos, 11 enunciados fueron acordes a las recomendaciones de las guías mientras que 2 enunciados estuvieron en desacuerdo y en los 4 restantes no pudo valorarse la concordancia debido a falta de respuesta unívoca en las guías.

\section{DISCUSIÓN}

Este trabajo representa la primera encuesta que mide el nivel de consenso y la aplicación de guías internacionales sobre el manejo de la diverticulitis aguda entre los miembros 
TABLA 6: TRATAMIENTO ELEGIDO SEGÚN HALLAZGO TOMOGRÁFICO

\begin{tabular}{|c|c|c|c|c|c|c|c|}
\hline $\begin{array}{l}\text { Clasificación } \\
\text { tomográfica * }\end{array}$ & & $\begin{array}{c}\text { Manejo } \\
\text { ambulatorio } \\
\%\end{array}$ & $\begin{array}{l}\text { Internación } \\
\text { y atb EV } \\
\%\end{array}$ & $\begin{array}{c}\text { Drenaje } \\
\text { percutáneo } \\
\%\end{array}$ & $\begin{array}{c}\text { Drenaje } \\
\text { y atb EV } \\
\%\end{array}$ & $\begin{array}{l}\text { Lavado } \\
\text { peritoneal } \\
\%\end{array}$ & $\begin{array}{l}\text { resección } \\
\text { quirúrgica } \\
\%\end{array}$ \\
\hline Estadio 0 & $\begin{array}{l}\text { Engrosamiento pared } \\
\text { colónica, aumento } \\
\text { densidad grasa pericolica }\end{array}$ & 23 & 77 & & & & \\
\hline Estadio la & $\begin{array}{l}\text { Burbujas de aire pericolico } \\
\text { o pequeña cantidad de } \\
\text { líquido pericolico sin a } \\
\text { bsceso dentro de } 5 \mathrm{~cm} \text { del } \\
\text { segmento inflamado }\end{array}$ & & 80,3 & & 13,1 & 4,9 & 1,7 \\
\hline Estadio lb & Absceso $\leq 4 \mathrm{~cm}$ & & 62,3 & 4,9 & 26,2 & 3,3 & 3,3 \\
\hline Estadio Ila & Absceso > 4cm & & & 19,7 & 63,9 & 11,5 & 4,9 \\
\hline Estadio IIb & $\begin{array}{l}\text { Aire distal }>5 \mathrm{~cm} \text { del } \\
\text { intestino inflamado }\end{array}$ & 1,6 & 59 & & 6,6 & 16,4 & 16,4 \\
\hline Estadio $3^{\star *}$ & $\begin{array}{l}\text { Líquido libre difuso sin aire } \\
\text { a distancia (sin perforación) }\end{array}$ & & & & & 41 & 59 \\
\hline Estadio $4^{\star *}$ & $\begin{array}{l}\text { Líquido libre difuso con aire } \\
\text { a distancia (perforación } \\
\text { persistente) }\end{array}$ & & & & & 41 & 59 \\
\hline
\end{tabular}

*WSES acute diverticulitis working group, ${ }^{* *}$ Ios estadios 3 y 4 se unificaron en la encuesta para evaluar el abordaje de la peritonitis y evitar diferencias de interpretación (cantidad de aire libre, localización, etc.).

TABLA 7: USO DE ANALGÉSICOS EN EL MANEJO AMBULATORIO DE LA DANC

$\begin{array}{lcc} & \mathrm{n}=61 & \% \text { Total } \\ \text { No usa } & 26 & 42,60 \% \\ \text { Rutinario } & 28 & 45,90 \% \\ \text { Selectivo } & 7 & 11,50 \%\end{array}$

TABLA 8: USO DE LOS ANTIBIÓTICOS EN EL MANEJO AMBULATORIO DE LA DANC

\begin{tabular}{|c|c|c|c|c|c|}
\hline & No usa & 7 días & 14 días & 21 días & $\%$ Total \\
\hline No usa & 3 & & & & $4,90 \%$ \\
\hline Rutinario & & 32 & 17 & & $80,30 \%$ \\
\hline $\begin{array}{l}\text { Selectivo (co- } \\
\text { morbilidades, } \\
\text { SIRS) }\end{array}$ & & 7 & 2 & & $14,80 \%$ \\
\hline $\begin{array}{l}\text { Uso rutinario } \\
7 \text { días y se- } \\
\text { lectivamente } \\
\text { extiendo a }\end{array}$ & & & 7 & 1 & $13,10 \%$ \\
\hline
\end{tabular}

TABLA 9: MANEJO DE LAS FALLAS TERAPÉUTICAS DE LOS TRATAMIENTOS NO RESECTIVOS EN LA DAC

\begin{tabular}{lccc} 
& $\begin{array}{c}\text { Resección } \\
\text { quirúrgica }\end{array}$ & $\begin{array}{c}\text { Lavado } \\
\text { peritoneal }\end{array}$ & $\begin{array}{c}\text { Drenaje } \\
\text { percutáneo }\end{array}$ \\
$\begin{array}{l}\text { Falla de drenaje } \\
\text { percutáneo }\end{array}$ & $56,70 \%$ & $33,30 \%$ & $10,00 \%$ \\
$\begin{array}{l}\text { Falla del lavado } \\
\text { peritoneal }\end{array}$ & $96,30 \%$ & $1,90 \%$ & $1,90 \%$ \\
\hline
\end{tabular}

de la SACP o entre cualquier otra agrupación médica a nivel nacional. Los resultados del mismo mostraron una amplia heterogeneidad en la indicación y elección de los estu-

TABLA 10: TIPO DE RESECCIÓN QUIRÚRGICA SEGÚN ESCENARIO

\begin{tabular}{|c|c|c|c|}
\hline $\begin{array}{l}\text { Tipo de resección } \\
\text { quirúrgica }\end{array}$ & $\begin{array}{l}\text { Peritonitis } \\
\text { purulenta }\end{array}$ & $\begin{array}{l}\text { Peritonitis } \\
\text { fecaloidea }\end{array}$ & $\begin{array}{l}\text { Cirugía } \\
\text { electiva }\end{array}$ \\
\hline Op. Hartmann & $46,70 \%$ & $77,40 \%$ & 0 \\
\hline Anastomosis primaria & $23,30 \%$ & $3,20 \%$ & $98,30 \%$ \\
\hline $\begin{array}{l}\text { Anastomosis primaria } \\
\text { con ostomía de } \\
\text { protección }\end{array}$ & $30 \%$ & $19,40 \%$ & $1,70 \%$ \\
\hline TOTAL & $100 \%$ & $100 \%$ & $100 \%$ \\
\hline
\end{tabular}

TABLA 11: DETALLES TÉCNICOS DE LA RESECCIÓN QUIRÚRGICA

\begin{tabular}{|c|c|c|}
\hline Detalles técnicos & $\begin{array}{l}\text { Urgencia } \\
n=61(\%)\end{array}$ & $\begin{array}{c}\text { Electiva } \\
\mathrm{n}=59(\%)\end{array}$ \\
\hline $\begin{array}{l}\text { Preparación mecánica sistemáti- } \\
\text { ca del intestino }\end{array}$ & $4(6,6)$ & $50(84,7)$ \\
\hline Abordaje laparoscópico de ser factible & $50(82)$ & $57(96,6)$ \\
\hline $\begin{array}{l}\text { Resección hasta márgenes sanos } \\
\text { de colon y recto }\end{array}$ & $53(86,9)$ & $53(89,8)$ \\
\hline Preservo arteria hemorroidaria superior & $5(8,2)$ & $13(22)$ \\
\hline $\begin{array}{l}\text { Desciendo el ángulo esplénico } \\
\text { sistemáticamente }\end{array}$ & $24(39,3)$ & $31(52,5)$ \\
\hline $\begin{array}{l}\text { Evaluación de fuga de la anasto- } \\
\text { mosis sistemática }\end{array}$ & $35(57,4)$ & $54(91,5)$ \\
\hline $\begin{array}{l}\text { Anastomosis primarias aún en perito- } \\
\text { nitis fecales, en condiciones óptimas }\end{array}$ & $15(24,6)$ & Nc \\
\hline
\end{tabular}

dios diagnósticos, del manejo terapéutico en la urgencia y del seguimiento luego del episodio agudo, logrando un nivel de consenso igual o mayor de $70 \%$ tan solo en 17 enunciados de los cuales solo 11 fueron acordes a las recomenda- 
TABLA 12: INDICACIÓN DE COLONOSCOPIA LUEGO DE RESPUESTA FAVORABLE

\begin{tabular}{|c|c|c|c|}
\hline Indicación & $\begin{array}{c}\text { Manejo } \\
\text { ambulatorio }\end{array}$ & $\begin{array}{c}\text { Drenaje } \\
\text { percutáneo }\end{array}$ & $\begin{array}{l}\text { Lavado } \\
\text { peritoneal }\end{array}$ \\
\hline Rutinaria & $38,30 \%$ & $29,80 \%$ & $20,40 \%$ \\
\hline $\begin{array}{l}\text { Primer episodio o no } \\
\text { tiene vcc reciente }\end{array}$ & $28,30 \%$ & $70,20 \%$ & $\mathrm{Nc}$ \\
\hline $\begin{array}{l}\text { No es necesaria, } \\
\text { seguir protocolo } \\
\text { pesquisa ccr }\end{array}$ & $33,30 \%$ & Nc & $\mathrm{Nc}$ \\
\hline
\end{tabular}

TABLA 13: INDICACIÓN DE CIRUGÍA ELECTIVA LUEGO DE RESPUESTA FAVORABLE

\begin{tabular}{|c|c|c|c|}
\hline & $\begin{array}{c}\text { Manejo } \\
\text { ambulatorio }\end{array}$ & $\begin{array}{c}\text { Drenaje } \\
\text { percutáneo }\end{array}$ & $\begin{array}{l}\text { Lavado } \\
\text { peritoneal }\end{array}$ \\
\hline $\begin{array}{l}\text { Consensuada } \\
\text { con el paciente }\end{array}$ & $\mathrm{Nc}$ & $61,00 \%$ & $\mathrm{Nc}$ \\
\hline Rutinaria & Nc & $3,40 \%$ & $48,10 \%$ \\
\hline $\begin{array}{l}\text { Selectiva (facto- } \\
\text { res del paciente) }\end{array}$ & $63,90 \%$ & $35,60 \%$ & $\mathrm{Nc}$ \\
\hline $\begin{array}{l}\text { Pacientes meno- } \\
\text { res de } 50 \text { años }\end{array}$ & $1,60 \%$ & $\mathrm{Nc}$ & $\mathrm{Nc}$ \\
\hline Nunca la indico & $34,40 \%$ & Nc & Nc \\
\hline
\end{tabular}

Nc: no corresponde, no se dio esa opción para esa pregunta.

$$
\text { Definición de sospecha de DA: }
$$

- Dolor abdominal y a la compresión en CII (WSES, EAES-SAGES).

-Ausencia de vómitos e historia previa de diverticulitis (Holanda).

-Proteina C reactiva $>50 \mathrm{mg} / \mathrm{L}$. (WSES).

\section{Protocolo diagnóstico y evaluación severidad}

-El diagnóstico clínico no es suficiente para realizar diagnóstico ni para indicar tratamiento. - Incorporar Prot. C reactiva: (EAES-SAGES).

-TC es el mejor estudio: (Dinamarca, ASCRS, Italia, WSES, Japón y EAES-SAGES).

Gráfico 1: Resumen de las Guías Internacionales, Diagnóstico y Severidad de DA.

\section{DANC, Hinchey 0-Ia}

-Ante SIRS o inmunocompromiso todas las guías indican antibióticos.

- Ante inmunocompetentes la gran mayoria aconseja omitir los antibióticos bajo seguimiento apropiado. El régimen ambulatorio es aceptable.

\section{Hinchey Ib-II}

- Los antibióticos son el tratamiento de primera línea para abscesos, el drenaje percutáneo se indica en abscesos grandes $(>3-4 \mathrm{~cm})$, en aquellos que no resuelven con antibióticos $y / 0$ en presencia de deterioro clínico.

- Cirugía de urgencia: está indicada cuando se hayan agotado los tratamientos no operatorios o sin mejoría del estado clínico. algunas guías indican explicitamente resección quirúrgica.

-Aire libre pericólico sin extravasación de contraste: en pacientes estables se indica antibióticos.

- Aire libre a distancia: En pacientes seleccionados inicialmente indicar antibióticos con estricto seguimiento para detectar fallas ter

\section{Hinchey III-IV}

- Todas las guías indican cirugía de urgencia como tratamiento de la peritonitis.

- Lavado peritoneal laparoscópico: si bien muchas guías lo desaconsejan las mas recientes la aceptan como tratamiento válido de Hinchey III en casos seleccionados y en manos entrenadas.

- Resección quirúrgica: Se recomienda en Hinchey IV por todas las guías. existe controversia sobre realizar o no anastomosis primaria con o sin ostomia de protección, en resumen en pacientes apropiados realizar anastomosis primaria y en pacientes inestables realizar operación de mann o cirugía de control de daños.

- Se recomienda limitar la resección al sector inflamado y no movilizar el ángulo esplénico si se decide realizar operación de Hartman

-Algunas guias recomiendan el abordaje laparoscópico en casos seleccionados, en manos entrenadas.

Gráfico 2: Resumen de las Guías Internacionales, Tratamiento según severidad, clasificación modificada de Hinchey.

ciones internacionales. Este hecho replica los hallazgos de otras encuestas a nivel internacional. Las posibles causas de
Prevención de recurrencias

- La recomendación mas frecuentemente mencionada es la indicación de dieta rica en fibras y si bien se han mencionado otras recomendaciones, ninguna cuenta con suficiente evidencia para parecer en todas las guías.

\section{Colonoscopia electiva}

- Aunque las recomendaciones de las guías generan confusión se puede decir al respecto que todas tienen como objetivo de descartar a existencia de un proceso neoplasico subyacente, y de indicar su realización en un periodo libre de inflamación.

- De modo que, ante una sospecha neoplasica importante la colonoscopia está claramente indicada, y si el paciente tiene (según protocolo de pesquisa de CCR) una colonoscopia reciente que descarte patología neoplásica no sería necesario repetir el estudio.

\section{Resección quirúrgica electiva}

-Su indicación depende de la severidad, la competencia inmunologica y la calidad de vida del paciente luego del episodio. Siendo claramente indicada en pacientes inmunocomprometidos

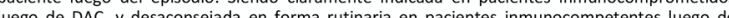
DANC.

- No hay indi

experiencia, la hasta tejido sano y por debajo de la union rectosigmoidea, movilizar el ángulo esplénico, realizar anastomosis siempre en inmunocompetentes y prueba de fuga anastomótica. sobre el nivel de sección vascular las recomendaciones no son concluyentes.

Gráfico 3: Resumen de las Guías Internacionales, Manejo luego de superado el episodio agudo.

TABLA 14: NIVEL DE RELEVANCIA DE LAS INDICACIONES PARA PREVENIR LAS RECURRENCIAS

\begin{tabular}{|c|c|}
\hline Indicaciones para prevención de recurrencias & $\begin{array}{l}\text { Relevancia } \\
\text { promedio }\end{array}$ \\
\hline $\begin{array}{l}\text { (1 definitivamente no relevante, } 9 \text { definitiva- } \\
\text { mente relevante) }\end{array}$ & $(1-9)$ \\
\hline Dieta rica en fibras & 7,4 \\
\hline Laxantes & 2,5 \\
\hline Evitar semillas & 2,8 \\
\hline Evitar AINES o aspirina & 3,2 \\
\hline Uso de mesalasina & 3,6 \\
\hline $\begin{array}{l}\text { Uso de rifaximina u otro antibióticos no ab- } \\
\text { sorbible }\end{array}$ & 4,6 \\
\hline Uso de probióticos & 4,3 \\
\hline Actividad física rutinaria & 6,1 \\
\hline
\end{tabular}

esta heterogeneidad a pesar de las múltiples guías publicadas deben buscarse en la interacción entre las publicaciones, las propias guías y los profesionales que las usan.

Respecto de las publicaciones se observa que la gran mayoría tiene un moderado a bajo nivel de evidencia y abundan los sesgos de selección, ya que existen pocos trabajos randomizados y muchos carecen de una clara pregunta que guíe la investigación. Otro aspecto importante es que en general son publicaciones de centros de referencia o hiper especializados, lo que dificulta lograr un nivel de validación externa adecuado, a modo de ejemplo, respecto del tratamiento quirúrgico en la urgencia. Recientemente se ha publicado un estudio retrospectivo con más de 10.000 pacientes que mostró diferencias muy significativas entre cirujanos generales y coloproctólogos respecto de morbimortalidad en la realización de resección con anastomosis primaria con ileostomía de protección en peritonitis diverticular, cuestionando la recomendación de intentar reconstruir el tránsito luego de la resección debido a los malos resul- 
TABLA 15: ENUNCIADOS CON CONSENSO Y APLICACION DE LAS GUÍAS

\begin{tabular}{|c|c|c|}
\hline & Porcentaje alcanzado & Acuerdo con las guías \\
\hline $\begin{array}{l}\text { El diagnóstico y la evaluación del paciente con DA requiere estudios } \\
\text { complementarios, no es suficiente el cuadro clínico. }\end{array}$ & 82 & $\mathrm{Si}$ \\
\hline La TC es el mejor estudio por imágenes. & 78,7 & $\mathrm{Si}$ \\
\hline La TC se debe solicitar con cte oral. & 72,1 & No evaluable \\
\hline La TC se debe solicitar con cte EV. & 88,5 & No evaluable \\
\hline $\begin{array}{l}\text { La DANC (estadio 0) se trata con antibióticos en forma rutinario } \\
\text { (sumado ambulatorio e internación). }\end{array}$ & 95,5 & No \\
\hline El manejo ambulatorio requiere antibióticos en forma rutinaria. & 80,3 & No \\
\hline $\begin{array}{l}\text { La presencia de Burbujas de aire pericolico o pequeña cantidad de } \\
\text { líquido pericolico sin absceso dentro de } 5 \mathrm{~cm} \text { del segmento inflamado } \\
\text { (estadio 1a) requiere internación y antibióticos EV. }\end{array}$ & 80,3 & $\mathrm{Si}$ \\
\hline Ante falla del LPL se indica resección quirúrgica. & 96,3 & No evaluable \\
\hline $\begin{array}{l}\text { Ante peritonitis fecaloidea el procedimiento de elección es la oper- } \\
\text { ación de Hartmann. }\end{array}$ & 77,4 & No evaluable \\
\hline $\begin{array}{l}\text { En cirugía electiva el procedimiento de elección es la anastomosis pri- } \\
\text { maria. }\end{array}$ & 98,3 & $\mathrm{Si}$ \\
\hline $\begin{array}{l}\text { En cirugía electiva es mandatorio la preparación mecánica sistemáti- } \\
\text { ca del intestino. }\end{array}$ & 84,7 & $\mathrm{Si}$ \\
\hline $\begin{array}{l}\text { En cirugía electiva se prefiere el abordaje laparoscópico en la resec- } \\
\text { ción de ser este factible. }\end{array}$ & 96,6 & $\mathrm{Si}$ \\
\hline $\begin{array}{l}\text { En cirugía electiva es mandatorio la resección hasta márgenes sanos } \\
\text { de colon y recto. }\end{array}$ & 89,8 & $\mathrm{Si}$ \\
\hline $\begin{array}{l}\text { En cirugía electiva es mandatorio la evaluación sistemática de fuga de } \\
\text { la anastomosis. }\end{array}$ & 91,5 & $\mathrm{Si}$ \\
\hline $\begin{array}{l}\text { En cirugía de urgencia se prefiere el abordaje laparoscópico en la re- } \\
\text { sección de ser este factible. }\end{array}$ & 82 & $\mathrm{Si}$ \\
\hline $\begin{array}{l}\text { En cirugía de urgencia es mandatorio la resección hasta márgenes sa- } \\
\text { nos de colon y recto. }\end{array}$ & 86,9 & $\mathrm{Si}$ \\
\hline $\begin{array}{l}\text { Indicación de colonoscopia luego de respuesta favorable a drenaje } \\
\text { percutáneo solo si es primer episodio o no tiene vcc reciente. }\end{array}$ & 70,2 & Si \\
\hline
\end{tabular}

tados en el grupo de cirujanos generales. ${ }^{18}$

Con respecto a las guías, si bien fue posible sintetizar las recomendaciones de las 8 guías publicadas en los últimos 10 años, fue notoria la disparidad de la metodología empleada en su elaboración a pesar de mencionar en su mayoría al sistema GRADE ${ }^{19-22}$ (Grading of Recommendations Assessment, Development and Evaluation) como método empleado. En este sistema las recomendaciones tienen como origen una pregunta clara que debiera incluir 4 componentes:

1. pacientes,

2. una intervención,

3. una comparación y

4. un resultado de interés.

A modo de ejemplo, en las guías más recientes las preguntas tienen esta estructura:

“CCuáles son las estrategias no quirúrgicas óptimas en el manejo de la DANC?”. ${ }^{14}$
“Es efectiva la terapia antibiótica para la diverticulitis sin abscesos o perforación?”. 13

Según GRADE sería:

¿Cuál es el efecto en:

1. pacientes inmunocompetentes con DANC de

2. la utilización de antibióticos

3. en comparación a no utilizarlos,

4. en términos de reducción de morbimortalidad, aceleración de la curación, progresión a abscesos o peritonitis, efectos adversos, resistencia antibiótica, disponibilidad y costos?

Existen además otros aspectos a tener en cuenta para determinar la fuerza de la recomendación. A saber:

1. el balance entre efectos deseables e indeseables,

2. la calidad de la evidencia,

3. valores y preferencias de la población (médicos y pacientes) y

4. costos (asignación de los recursos). 
Estos últimos puntos dificultan la extrapolación de guías internacionales a nuestro ámbito local.

\section{CONCLUSIÓN}

Si consideramos la baja evidencia de los trabajos científicos, la pobre metodología empleada en las guías internacionales para elaborar preguntas y recomendaciones y la necesidad de contar con datos locales sobre costos y preferencias, se hace evidente la necesidad que en nuestro ámbito se comience a investigar en forma organizada, sea desde nuestra institución u otra, con el objetivo de avanzar hacia la realización de una guía nacional que contem- ple las realidades y recursos de cada región, con el objetivo último de convalidar a través de un consenso las recomendaciones para un manejo adecuado de la diverticulitis en nuestro medio.

\section{AGRADECIMIENTOS}

Primeramente, agradezco el acompañamiento y consejo del Dr. Pablo Piccinini quien gentilmente accedió a ser mi tutor en la realización de este trabajo. De igual modo, extiendo las gracias a los Dres. Luis Pedro y Cristian Rodríguez, por sus valiosos consejos.

\section{BIBLIOGRAFÍA}

1. de Korte N, Klarenbeek BR, Kuyvenhoven JPh, Roumen RMH, Cuesta MA, Stockmann HBAC. Management of diverticulitis: results of a survey among gastroenterologists and surgeons: Management of diverticulitis: results of a survey among gastroenterologists and surgeons. Colorectal Dis. diciembre de 2011;13(12):e411-7.

2. O'Leary DP, Lynch N, Clancy C, Winter DC, Myers E. International, Expert-Based, Consensus Statement Regarding the Management of Acute Diverticulitis. JAMA Surg. 1 de septiembre de 2015;150(9):899.

3. Jaung R, Robertson J, Rowbotham D, Bissett I. Current management of acute diverticulitis: a survey of Australasian surgeons. 2016;129(1431):8.

4. Siddiqui J, Zahid A, Hong J, Young CJ. Colorectal surgeon consensus with diverticulitis clinical practice guidelines. World J Gastrointest Surg. 27 de noviembre de 2017;9(11):224-32.

5. Andersen JC, Bundgaard L, Elbrønd H, Laurberg S, Walker LR, Støvring J. Danish national guidelines for treatment of diver- ticular disease. 2012;12.

6. Andeweg CS, Mulder IM, Felt-Bersma RJF, Verbon A, van der Wilt GJ, van Goor H, et al. Guidelines of Diagnostics and Treatment of Acute Left-Sided Colonic Diverticulitis. Dig Surg. 2013;30(46):278-92.

7. Feingold D, Steele SR, Lee S, Kaiser A, Boushey R, Buie WD, et al. Practice Parameters for the Treatment of Sigmoid Diverticulitis: Dis Colon Rectum. marzo de 2014;57(3):284-94.

8. Cuomo R, Barbara G, Pace F, Annese V, Bassotti G, Binda GA, et al. Italian consensus conference for colonic diverticulosis and diverticular disease. United Eur Gastroenterol J. octubre de 2014;2(5):413-42.

9. Stollman N, Smalley W, Hirano I, Adams MA, Dorn SD, DudleyBrown SL, et al. American Gastroenterological Association Institute Guideline on the Management of Acute Diverticulitis. Gastroenterology. diciembre de 2015;149(7):1944-9.

10. Sartelli M, Catena F, Ansaloni L, Coccolini F, Griffiths EA, AbuZidan FM, et al. WSES Guidelines for the management of acute left sided colonic diverticulitis in the emergency setting. World J Emerg Surg. diciembre de 2016;11(1):37.

11. Klarenbeek BR, de Korte N, van der Peet DL, Cuesta MA. Review of current classifications for diverticular disease and a translation into

clinical practice. Int J Colorectal Dis. febrero de 2012;27(2):207-14.

12. Sartelli M, Moore FA, Ansaloni L, Di Saverio S, Coccolini F, Griffiths EA, et al. A proposal for a CT driven classification of left colon acute diverticulitis. World J Emerg Surg. 19 de febrero de 2015;10(1):3.

13. Nagata N, Ishii N, Manabe N, Tomizawa K, Urita $Y$, Funabiki $T$, et al. Guidelines for Colonic Diverticular Bleeding and Colonic Diverticulitis: Japan Gastroenterological Association. Digestion. 2019;99(1):1-26.

14. Francis NK, Sylla P, Abou-Khalil M, Arolfo S, Berler D, Curtis NJ, et al. EAES and SAGES 2018 consensus conference on acute diverticulitis management: evidence-based recommendations for clinical practice. Surg Endosc. septiembre de 2019;33(9):2726-41.

15. Hardigan PC, Popovici I, Carvajal MJ. Response rate, response time, and economic costs of survey research: A randomized trial of practicing pharmacists. Res Soc Adm Pharm. 1 de enero de 2016;12(1):141-8

16. Reinisch JF, Yu DC, Li W-Y. Getting a Valid Survey Response From 662 Plastic Surgeons in the 21st Century: Ann Plast Surg. enero de 2016;76(1):3-5.

17. Manfreda KL, Bosnjak M, Berzelak J, Haas I, Vehovar V. Web Surveys versus other Survey Modes: A Meta-Analysis Comparing Response Rates. Int J Mark Res. enero de 2008;50(1):79-104.

18. Goldstone RN, Cauley CE, Chang DC, Kunitake H, Ricciardi $\mathrm{R}$, Bordeianou L. The Effect of Surgical Training and Operative Approach on Outcomes in Acute Diverticulitis: Should Guidelines Be Revised? Dis Colon Rectum. enero de 2019;62(1):71-8.

19. Guyatt GH, Oxman AD, Vist GE, Kunz R, Falck-Ytter Y, AlonsoCoello P, et al. GRADE: an emerging consensus on rating quality of evidence and strength of recommendations. BMJ. 24 de abril de 2008;336(7650):924-6.

20. Guyatt GH, Oxman AD, Kunz R, Vist GE, Falck-Ytter Y, Schünemann HJ. What is "quality of evidence" and why is it important to clinicians? BMJ. 1 de mayo de 2008;336(7651):995-8.

21. Guyatt GH, Oxman AD, Kunz R, Falck-Ytter Y, Vist GE, Liberati A, et al. Going from evidence to recommendations. BMJ. 8 de mayo de 2008;336(7652):1049-51.

22. Guyatt GH, Oxman AD, Kunz R, Jaeschke R, Helfand M, Liberati $\mathrm{A}$, et al. Incorporating considerations of resources use into grading recommendations. BMJ. 22 de mayo de 2008;336(7654):1170-3.

\section{COMENTARIO}

La prevalencia de la enfermedad diverticular ha sufrido un constante aumento durante las últimas décadas. Probablemente por el desarrollo y perfeccionamiento de las técnicas diagnósticas por un lado y del envejecimiento de la población por el otro. Su abordaje terapéutico también mutó en este periodo, generándose en forma constante controversias respecto a las premisas que hasta hace unos años pocos se animaban a cuestionar. 
El presente trabajo describe los resultados de una encuesta nacional que refleja la falta de consenso que existe en nuestro medio sobre varios aspectos terapéuticos de la enfermedad. Hecho que también sucede a nivel internacional. Cabe destacar, en contraste a lo que se recomienda en las guías internacionales, que en la Argentina se indica con mayor frecuencia en forma rutinaria el uso de antibióticos ante enfermedad diverticular no complicada. Por otro lado, dentro de la heterogeneidad de los resultados de la misma, es notorio que cada vez más profesionales consideran como opción el lavado peritoneal y la anastomosis primaria con o sin protección frente a la diverticulitis complicada y fracaso del tratamiento no quirúrgico.

Sebastián Guckenheimer Hospital Dr. I. Pirovano, C.A.B.A., Argentina. 